\title{
General radiographic attributes of optically stimulated luminescence dosimeters: a basic insight
}

\begin{abstract}
We report the ubiquitous radiographic characteristics of optically stimulated luminescence dosimeters (OSLD) so called nanoDot OSLDs (Landauer Inc., Glendwood, IL). The X-ray irradiations were performed in free air ambiance to inspect the repeatability, the reproducibility, the signal depletion, the element correction factors (ECFs), the dose response and the energy dependence. Repeatability of multiple readouts after single irradiation to $10 \mathrm{mGy}$ revealed a coefficient of variation below $3 \%$, while the reproducibility in repeated irradiation-readout-annealing cycles was above $2 \%$. The OSL signal depletion for three nanoDots with simultaneous irradiation to $20 \mathrm{mGy}$ and sequential readouts of 25 times displayed a consistent signal reduction $\approx 0.5 \%$ per readout with $\mathrm{R} 2$ values over 0.98 . ECFs for individual OSLDs were varied from 0.97 to 1.03 . In the entire dose range under $80 \mathrm{kV}$, a good linearity with an R2 exceeding 0.99 was achieved. Besides, the percentage difference between OSLD and ion-chamber dose was less than 5\%, which was superior to TLD. The X-ray photon irradiated energy response factors (between 0.76 and 1.12) in the range of $40-150 \mathrm{kV}$ (26.1-61.2 keV) exhibited significant energy dependence. Indeed, the nanoDot OSLDs disclosed good repeatability, reproducibility and linearity. The OSLDs measured doses were closer to ion-chamber doses than that of TLD. It can be further improved up to $\approx 3 \%$ by applying the individual dosimeter ECF. On top, the energy dependent uncertainties can be minimized using the energy correction factors. It is established that the studied nanoDot OSLDs are prospective for measuring entrance dose in general radiographic practices.
\end{abstract}

Keyword: Radiography; Nano Dot; X-ray; OSL dosimeter; MicroStar 
